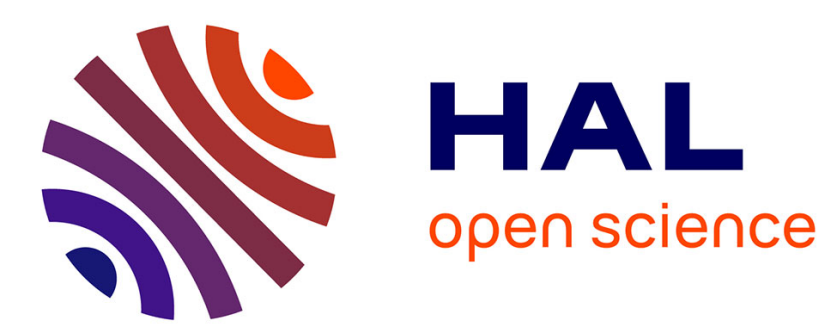

\title{
An agent-based model of dune interactions produces the emergence of patterns in deserts
}

\author{
Mathieu Génois, Sylvain Courrech Du Pont, Pascal Hersen, Guillaume
}

Grégoire

\section{- To cite this version:}

Mathieu Génois, Sylvain Courrech Du Pont, Pascal Hersen, Guillaume Grégoire. An agent-based model of dune interactions produces the emergence of patterns in deserts. Geophysical Research Letters, 2013, 40, pp.3909. 10.1002/grl.50757 . hal-00759289v2

\section{HAL Id: hal-00759289 \\ https://hal.science/hal-00759289v2}

Submitted on 30 Jun 2016

HAL is a multi-disciplinary open access archive for the deposit and dissemination of scientific research documents, whether they are published or not. The documents may come from teaching and research institutions in France or abroad, or from public or private research centers.
L'archive ouverte pluridisciplinaire HAL, est destinée au dépôt et à la diffusion de documents scientifiques de niveau recherche, publiés ou non, émanant des établissements d'enseignement et de recherche français ou étrangers, des laboratoires publics ou privés. 


\title{
An agent-based model of dune interactions produces the emergence of patterns in deserts
}

\author{
Mathieu Génois, ${ }^{1}$ Sylvain Courrech du Pont, ${ }^{1}$ Pascal Hersen, ${ }^{1}$ and Guillaume Grégoire ${ }^{1,2}$ \\ Received 13 June 2013; revised 9 July 2013; accepted 12 July 2013; published 7 August 2013.
}

[1] Crescent-shaped barchan dunes are highly mobile dunes which are ubiquitous on Earth and other solar system bodies. Although they are unstable when considered separately, they form large assemblies in deserts and spatially organize in narrow corridors that extend in the wind direction. Collision of barchans has been proposed as a mechanism to redistribute sand between dunes and prevent the formation of very large dunes. Here we use an agent-based model with elementary rules of sand redistribution during collisions to access the full dynamics of very large barchan fields. We tune the dune field density by changing the sand load/lost ratio and follow the transition between dilute fields, where barchans barely interact, and dense fields, where dune collisions control and stabilize the dune field. In this dense regime, barchans have a small, well-selected size and form flocks: the dune field self-organizes in narrow corridors of dunes, as it is observed in real dense barchan deserts. Citation: Génois M., S. Courrech du Pont, P. Hersen, and G. Grégoire (2013), An agent-based model of dune interactions produces the emergence of patterns in deserts, Geophys. Res. Lett., 40, 3909-3914, doi:10.1002/grl.50757.

\section{Introduction}

[2] Depending on the variability of the local winds and sand availability, dunes can adopt various shapes [Wasson and Hyde, 1983]. When viewed from above, they mimic large stars, long linear ridges, or crescent structures [Bagnold, 1941; Pye and Tsoar, 1990]. The crescent-shaped dune, called barchan, is a prototypical model of sand dune dynamics, and its properties as an isolated object are now well understood [Bagnold, 1941; Finkel, 1959; Kroy et al., 2002; Hersen, 2004]. However, barchans are usually found in large dune assemblies, counting typically tens of thousands of dunes [Elbelrhiti et al., 2008; Bagnold, 1941; Cooke et al., 1993]. Barchan fields are observed in regions with a nonerodible floor and submitted to a prevalent unidirectional fluid flow. These conditions are often met, and barchan fields are observed on Earth, Mars, Titan, and even underseas [Breed et al., 1979; Bourke and Goudie, 2009; Radebaugh

Additional supporting information may be found in the online version of this article.

${ }^{1}$ Laboratoire Matière et Systèmes Complexes, University of Paris Diderot, Paris, France.

${ }^{2}$ Academy of Bradylogists (http://bradylogist.info/).

Corresponding author: S. Courrech du Pont, Laboratoire Matière et Systèmes Complexes, Université Paris-Diderot, Bâtiment Condorcet, 10 rue A. Domon et Léonie Duquet, 75205 Paris CEDEX 13, France. (sylvain.courrech@univ-paris-diderot.fr)

(C)2013. American Geophysical Union. All Rights Reserved. 0094-8276/13/10.1002/grl.50757 et al., 2010]. Barchan width ranges from a few meters to several hundreds of meters. They are usually close to each other, and thus, their dynamics, and in particular the evolution of their size through time, may be altered by dune-dune interactions. This has to be taken into consideration if one wants to understand the long-term evolution of barchan fields. In particular, the very existence of a barchan field is in apparent contradiction with the fact that barchan dynamics display an unstable fixed point. Dunes will either grow or shrink if their size departs from their equilibrium size, which is set by the balance between sand loss and sand capture. In contrast with this unstable behavior, dune size distribution within a field does not display a lack of small dunes or an anomalous number of huge barchans [Elbelrhiti et al., 2008; Durán et al., 2009, 2011]. Furthermore, barchan fields may spatially be structured in narrow corridors, which extend in the wind direction. As observed on Figure 1, these corridors organize the dune field in zones of dense barchan areas, where dunes are smaller, and dilute areas with larger dunes. Dense and sparse regions coexist nearby on the same field without any obvious reason, neither the local conditions such as wind velocity or grain size, nor the boundary conditions differ [Elbelrhiti et al., 2008]. This spatial organization is strongly different from a simple field confinement or barchan alignment [Bagnold, 1941], i.e., the classical diagonal or echelon arrangement, which can be simulated considering remote exchange of sand only as shown by Lima et al. [2002].

[3] A coexistence in time and in space of these regions of different barchan densities and sizes could be interpreted as the signature of a self-organized system. Sediment transport often leads to the formation of self-organized patterns like sand seas or watersheds [Rodriguez-Iturbe and Rinaldo, 1997; Dodds and Rothman, 2000; Devauchelle et al., 2012]. However, barchans are very mobile objects, which gives them a character of their own. The dynamical clusters they form on the field are, to some extent, reminiscent of collective movements as observed for schools of fish or flocks of birds. It is thus natural to use the formalism and the tools of this study field [Vicsek et al., 1995; Chaté et al., 2008], to consider barchans as agents, which interact through collisions. Indeed, it is commonly thought that dune-dune interactions, such as collisions, are at play to sustain a dune field over a long time, to structure the field in corridors and to select the dune size [Kocurek et al., 2010]. Field studies [Elbelrhiti et al., 2005; Hugenbholtz and Barchyn, 2012; Vermeesch, 2011] and underwater experiments [Endo et al., 2004; Hersen and Douady, 2005] have shown that dunes do exchange sand during collision events. Dune collision lead to merging or splitting mechanisms, which can be stabilizing providing that large dunes are regularly split into smaller ones, as proposed using a mean field approach 


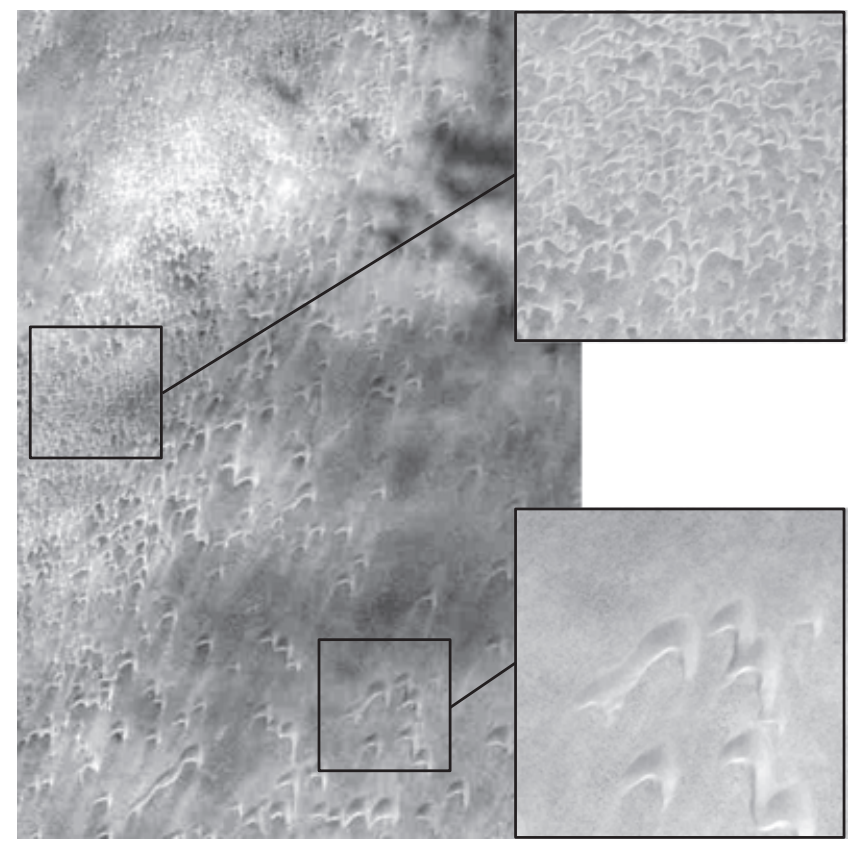

Figure 1. La'ayoune desert in south of Morocco $\left(27.3^{\circ} \mathrm{N}\right.$, $-13.21^{\circ} \mathrm{E}$ ), in which (top left) dense regions coexist with (bottom right) sparse regions. Square zooms are $1 \mathrm{~km}$ wide. (from Google Earth).

[Hersen and Douady, 2005]. This idea is only a first step to fully understand how collisions set the structure and affect the stability of a large assembly of dunes. Numerical studies implementing phenomenological rules of collisions have been run in order to forecast the statistical properties of a large dune field in which collisions take place [Durán et al., 2009]. The barchan size selection successfully emerges from these numerical models but not the critical aspect of spatial organization of dunes in corridors. It is a general problem in which the large-scale emergent property comes from the complex, local, interactions of many objects, which perfectly falls within the scope of an agent-based model.

\section{A Barchan Field as an Agent Population}

[4] Here we use an agent-based model, implementing dune collisions over the whole dune field, to infer the dynamical statistical behavior of a large dune field in the limit of long observation time, its stationary state and the possible emergence of spatial structures. In order to identify the physical mechanisms involved in the emergent properties of the dune fields, this model is restricted to the minimum ingredients of dune dynamics and interactions.

[5] The barchan shape is characterized by a low-slope upwind back and an avalanche face downwind, which is framed by two horns pointing in the wind direction (see Figures $2 \mathrm{a}-2 \mathrm{~b}$ ). The width, length, and height of barchans are linearly related to each other so that their morphological state can be defined by one parameter only. Sand erosion and deposition force barchans to move downwind. Their propagation velocity, which increases with the wind shear stress and decreases with the sediment influx, is inversely proportional to the dune size. The avalanche face acts as a sand trap, and barchans can propagate over long distances without losing much sand. Yet small sand loss occurs at the tip of the barchan horns [Bagnold, 1941; Pye and Tsoar, 1990]. The resulting output sand flux can be described by a linear relationship with the dune size. Starting with a nonnull value, the sand loss increases only very weakly with increasing dune size, and can be considered as a constant [Hersen et al., 2004]. On the other hand, the input sand flux is proportional to the dune width. As a result, the fixed point (where loss and gain are balanced) is unstable and an isolated barchan can only grow or shrink and disappear [Hersen et al., 2004]. Indeed, below a critical size, the barchan loses its avalanche face, turns into a dome-like structure, and quickly vanishes.

[6] In our model, dunes are described by their width $w$ only and, for the sake of simplicity, are cubic. They propagate downwind at a speed $v$ :

$$
v=\frac{\alpha}{w},
$$

where $\alpha$ is a volumic sediment flux per unit of length. It accounts for the wind speed and the sand transport threshold. We assume that dunes lose sand homogeneously along their downwind face. We call $\Phi$ the volume lost per unit of time because of wind erosion. Barchans can also grow, due to an incoming sand influx per unit of length transverse to the wind, $q_{\mathrm{a}}$. The volume $V$ of an isolated dune will then vary in function of time as:

$$
\frac{d V}{d t}=-\Phi+q_{\mathrm{a}} w
$$

Equation (2) contains the fundamental instability of one isolated barchan of unstable equilibrium size $\tilde{w}=\Phi / q_{\mathrm{a}}$. If the dune shrinks below the dome size $w_{c}$, it is removed from the field. The model does not conserve mass.

[7] One hypothesis to reconcile the unstable behavior of an isolated dune (equation (2)) with the existence of dense barchan fields is to consider dune collisions. Small dunes propagate faster than large ones (equation (1)) and can collide with them, leading to a transfer of mass between dunes [Hersen and Douady, 2005; Durán et al., 2009]. This mass exchange is not fully understood yet. However, numerical studies [Schwämmle and Herrmann, 2003; Katsuki et al., 2005; Diniega et al., 2010], experiments under water [Endo et al., 2004; Hersen and Douady, 2005], and field observations [Elbelrhiti et al., 2005; Hugenbholtz and Barchyn, 2012] have shown that barchan collisions lead to merging and splitting depending on the relative size of dunes and their lateral alignment. If the incoming dune is very small, it is simply absorbed by the larger, slower one. If the dunes are of similar sizes, a redistribution of mass occurs, and one (or several) small dunes are emitted at the front, while a larger dune is formed at the back.

[8] In our model, two dunes are considered in interaction if they are closer than a distance $d_{0}$ in the wind direction and if their width projections overlap, as shown in Figure $2 b$. We consider two types of interactions: a distant one through capture of emitted sand and sand flux screening, and a close one through collision. The distance $d_{0}$ reflects a typical distance for the sand flux to get diluted laterally due to sand grains collisions on the ground, equivalent to a diffusion process. Let us consider two dunes $i$ and $j, i$ being the downwind dune and $j$ the upwind one. The width of $i$ is noted $w_{i}$ and the overlapped width $\sigma_{i j}$. We define the upward projection $s=\frac{\sigma_{i j}}{w_{j}}$ and the downward projection $r=\frac{\sigma_{i j}}{w_{i}}$. The leeward dune catches a part $s$ of the sand lost by the upwind dune. 


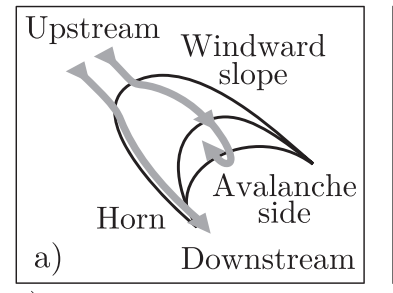

c)

$r<\varepsilon_{m} \quad r \geq \varepsilon_{m}$

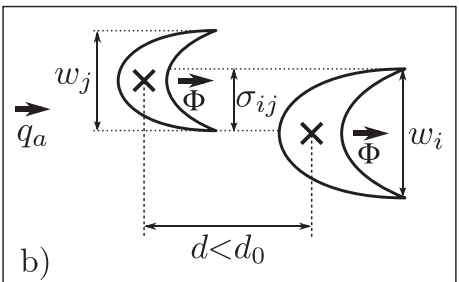

b)

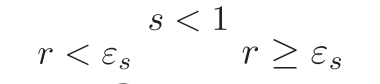

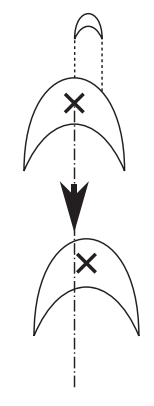

Merging

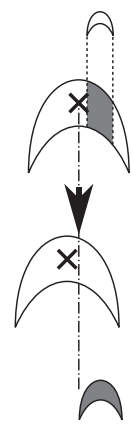

Exchange

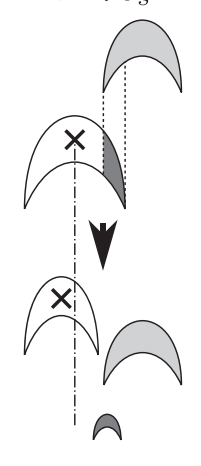

Fragmentation

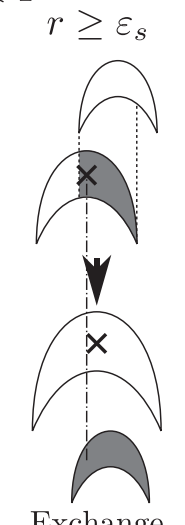

Exchange
Figure 2. Elementary rules for barchan field modeling (a) Morphology of a barchan. (b) Parameters used to describe an interaction: two dunes of size $w_{i}$ and $w_{j}$ interact when they are closer than $d_{0}$ and within a cross section of $\sigma_{i j}$. Each dune loses a volume per unit of time $\Phi$, and it is fed by a sand influx $q_{a}$. (c) The four cases of binary dune collisions. Depending on the upward projection $s=\frac{\sigma_{i j}}{w_{j}}$, the collision is total $(s=1)$ or lateral $(s<1)$. Then, the downward projection $r=\frac{\sigma_{i j}}{w_{i}}$ regarding to a merging $\varepsilon_{m}$ or a splitting $\varepsilon_{s}$ threshold sets the type of the collision. The volume of sand is conserved during a collision. Grey levels show the sand redistribution.

In the same time, the upwind dune screens the leeward dune on the width $\sigma_{i j}$ from any flux coming upwind of $i$ and $j$. If the upwind dune $j$ is the only one that is closer than $d_{0}$ to the downwind dune $i$, the volume of the latter varies as $d V_{i} / d t=s \times \Phi+q_{a}\left(w_{i}-\sigma_{i j}\right)-\Phi$. Note that in our model, these eolian mass exchanges do not affect the aspect ratio of the dune (cubic) nor their position. This simplification, along with a sand loss spread over the full dune width (not limited to the horns), prevent the barchans to align in echelons.

[9] Two dunes collide when the upwind one passes the downwind one, following the rules shown in Figure 2c. When $s=1$ (perfect overlap), $r$ is the width ratio. The two dunes merge if $r$ is smaller than the merging threshold $\varepsilon_{\mathrm{m}}$, and the new dune gets a volume: $V_{i}^{t+\Delta t}=\left(w_{i}^{t}\right)^{3}+\left(w_{j}^{t}\right)^{3}$. When $s=1$ and $r \geqslant \varepsilon_{\mathrm{m}}$, the total sand is redistributed into two new dunes of volume: $V_{i}^{t+\Delta t}=\left(w_{i}^{t}\right)^{3}+\left(w_{j}^{t}\right)^{3}-\sigma_{i j} w_{i}^{2}$ and $V_{j}^{t+\Delta t}=\sigma_{i j} w_{i}^{2}$. When $s<1$ (partial overlap), the sand is redistributed into two or three dunes, respectively, for $r$ values bigger or smaller than the splitting threshold $\varepsilon_{\mathrm{s}}$. When $s<1$ and $r \geqslant \varepsilon_{\mathrm{s}}$, the two dunes exchange sand the same way as when $s=1$ and $r \geqslant \varepsilon_{\mathrm{m}}$. When $s<1$ and $r<\varepsilon_{\mathrm{s}}$, the bumping dune $i$ is unaffected while the bumped dune is split in two dunes. The ejected dune $k$ gets a volume: $V_{k}^{t+\Delta t}=\sigma_{i j} w_{i}^{2}$ while $V_{j}^{t+\Delta t}=\left(w_{j}^{t}\right)^{3}$ and $V_{i}^{t+\Delta t}=\left(w_{i}^{t}\right)^{3}-\sigma_{i j} w_{i}^{2}$. Here the new $i$ and $j$ dunes at $t+\Delta t$ may be the upwind or downwind dunes depending on their size, the smaller being the new downwind dune. Note that the center of mass of the new dunes are set at the barycentric positions of the incoming sand, which may shift the dunes laterally.

[10] To compensate for sand loss, dunes can appear by nucleation anywhere in the dune field with a probability per unit time and per unit of surface $\lambda$. Their width is arbitrarily set to $w_{0}$. These nucleations are the trace of topographical defects that promote sand deposition [Pye and Tsoar, 1990; Bagnold, 1941]. This choice maximizes the effect of noise. Thus, any emerging behaviors will be robust.

[11] Looking for stationary behavior of large dune fields, the dune field boundaries are periodic and the field is long compared to the typical distance of dune interactions $\left(L \gg d_{0}\right)$. Since dunes move along the wind direction, we expect that the perpendicular direction $l$ does not play a major role in dune-dune dynamics. The field is initially filled with dunes at random and homogeneously chosen positions. Their size follow a constant probability which is centered on $w_{0}$ and with a minimum cutoff value $w_{\mathrm{c}}$. The numerical method used to compute the assembly of dunes in this large field is based on a synchronous algorithm and off-lattice dynamics as in self-propelled particle models [Chaté et al., 2008]. By their nontrivial kinematics, dunes can indeed be considered as self-propelled particles, which exchange mass - or momentum - with their neighborhood. Values of the different parameters used in the simulations are reported in Table 1. In particular, we assume that there is little sand around the dunes, so that the ambient influx is null $\left(q_{\mathrm{a}}=0\right)$. It implies that an isolated dune always vanishes. From a simulation to another, the dune density of the field is tuned through a fixed nucleation rate $\lambda$ and a changing erosion rate $\Phi$.

[12] Although variables such as $\alpha, q_{\mathrm{a}}, \Phi$, or $\lambda$ are partly physically linked (in a nontrivial manner), by the wind magnitude, for example, they are decoupled in our model. This helps to identify the physical ingredients that pilot the emergence of structures. The decoupling of the variables implies that it will be artificial to fix accurately their values with real figures. However, if dunes of minimum width $w_{c}$ correspond to $\sim 1 \mathrm{~m}$ dunes in real fields, they travel about 10 times their width length in a year time [see Cooke et al., 1993]. This gives an order of magnitude for dune sizes and velocities in our simulations (see Table 1).

\section{Results and Discussion. Dense Dune Fields Auto-Organize in Corridors.}

[13] From the model parameters, seven independent dimensionless numbers can be built. There are three ratios of lengths (for example, $w_{0} / d_{0}, w_{\mathrm{c}} / d_{0}$, and $\tilde{w} / d_{0}=\Phi /\left(q_{\mathrm{a}} d_{0}\right)$, which is infinite here), the two parameters $\varepsilon_{\mathrm{s}}$ and $\varepsilon_{\mathrm{m}}$, which control the dynamics of collisions and 2 times ratios. These

Table 1. Parameters of the Simulations

\begin{tabular}{cccccc}
\hline$d_{0}$ & $w_{0}$ & $w_{\mathrm{c}}$ & $\ell$ & $L$ & $\Delta t$ \\
\hline 1 & 0.1 & 0.01 & 32 & {$[32 ; 128]$} & 1 \\
\hline$\varepsilon_{\mathrm{s}}$ & $\varepsilon_{\mathrm{m}}$ & $\lambda^{-1}$ & $\alpha$ & $\Phi \times 10^{7}$ & $q_{\mathrm{a}}$ \\
\hline 0.5 & 0.5 & 2048 & $10^{-3}$ & {$[1.5 ; 500]$} & 0 \\
\hline
\end{tabular}


(a)

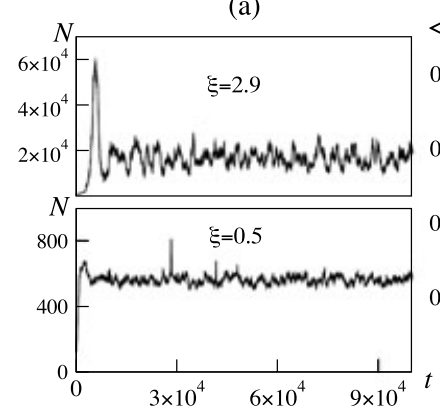

(c)
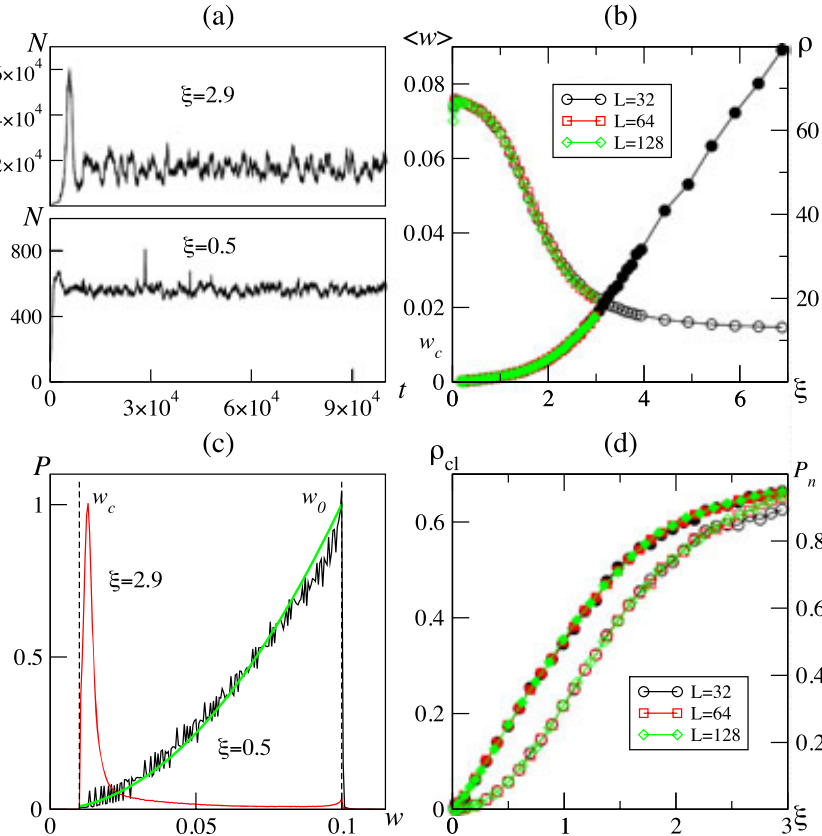

(d)

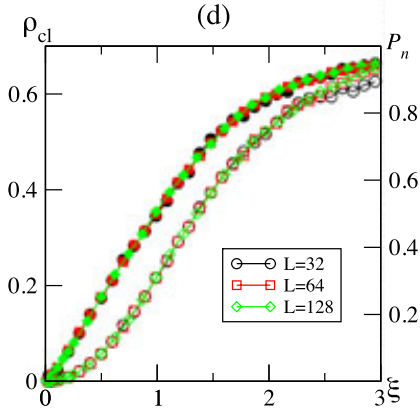

Figure 3. Characteristics of dilute and dense dune field. (a) Number of dunes in the field as a function of time for a dense (top) and dilute (bottom) regime. (b) Mean width $<w>$ (empty symbols) and dune density $\rho$ ( $\rho=N /(L \ell)$, filled symbols) in the field versus $\xi$ for different system lengths. (c) Size distribution of dunes in a dilute (red) and in a dense (black) regime field. The continuous green line is the analytic law of dilute field distribution, equation (4). (d) Density of clusters $\rho_{\mathrm{cl}}$ within the field (empty symbols) and probability $P_{n}$ for a dune to be in a cluster (filled symbols) versus $\xi$ for different system sizes.

last two dimensionless numbers are set with three relevant times which we identified as the time of disappearance of an isolated dune of size $w_{0}: t_{\text {eol }}=\left(w_{0}^{3}-w_{\mathrm{c}}^{3}\right) / \Phi$, the typical nucleation time: $t_{\text {nuc }}=\left(\lambda \times d_{0}^{2}\right)^{-1}$ and a collision time: $t_{\text {col }}$, which could be evaluated as the shortest time for two dunes to collide when they are under influence: $t_{\text {col }}=d_{0} /\left(\alpha / w_{\mathrm{c}}-\alpha / w_{0}\right)$. In the present study, all parameters but $\Phi$, the sand loss per unit time of a barchan, are kept constant (see Table 1): $t_{\text {nuc }}=2048$ and $t_{\text {col }} \simeq 11$ while $t_{\text {eol }} \in\left[20 ; 6.7 \times 10^{3}\right]$. An isolated dune of size $w_{0}$ travels a distance 2 to 660 times its initial size before disappearing. We define the control parameter $\xi$ :

$$
\xi=\frac{t_{\mathrm{eol}}}{t_{\mathrm{nuc}}}=\frac{w_{0}^{3}-w_{\mathrm{c}}^{3}}{\Phi} \lambda d_{0}^{2} .
$$

It measures the balance between the disappearance of dunes due to sand loss (function of $\Phi$ ) and dune nucleation (fixed).

[14] Although out of equilibrium, the dune field always reaches a stationary state within our range of parameters (see Figure 3a). For small $\xi$, the barchan field is dilute, a few dunes are dispersed across the whole field, the density is very low, and dune collisions are rare. Dunes can be considered as separate, unstable objects whose disappearance is balanced by nucleation only. $\xi$ is actually the exact dimensionless stationary density in the limit of $\xi \ll 1$. The normalized distribution of size $P(w)$ (see Figure 3c) follows the analytic distribution:

$$
P(w)=\frac{3 w^{2}}{w_{0}^{3}-w_{\mathrm{c}}^{3}}, \text { for } w \in\left[w_{\mathrm{c}} ; w_{0}\right],
$$

which can be derived from the individual dynamics (equation (2)). Therefore, the typical size of dune is about $3 / 4 w_{0}$, when $w_{c}$ is small enough. Such a field with low interaction between dunes compares well to dilute deserts such as the barchan field of La Pampa de la Joya in Peru [Elbelrhiti et al., 2008].

[15] Decreasing the sand loss $\Phi$, and thus increasing $\xi$, increases the frequency of collisions. For large densities $(\xi \geq 1)$, dune collisions tend to increase the number $N$ of dunes in the field more rapidly than the effect of nucleation itself as shown on Figure $3 \mathrm{~b}$. With $\varepsilon_{\mathrm{s}}$ and $\varepsilon_{\mathrm{m}}$ set to 0.5 , the effective predominant collision type is the fragmenting one that creates an additional dune (Figure 2c). These collisions dispatch sand in a nontrivial manner. The field state remains stationary (Figure $3 a$ ) but the size distribution is fundamentally altered and shifted to small sizes (Figures $3 b$ and $3 \mathrm{c}$ ). The most probable size is close to but larger than the minimum size of dunes $w_{\mathrm{c}}$ (Figure $3 \mathrm{c}$ ). A dense assembly of dunes is not a trivial homogeneous field with frequent (a)

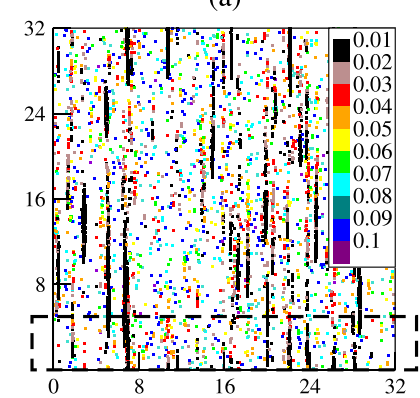

(c)

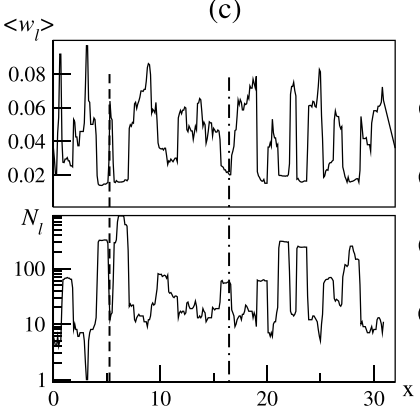

(b)

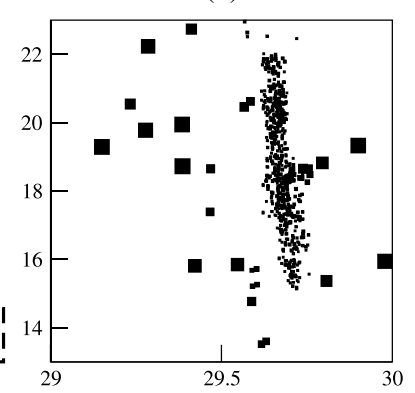

(d)

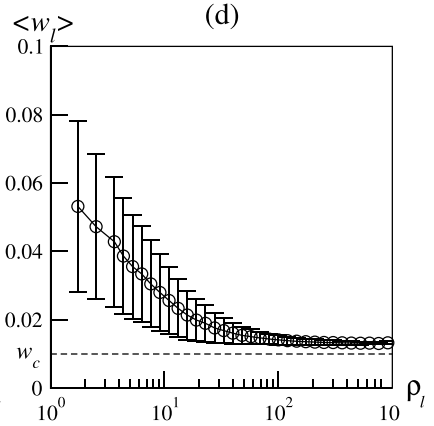

Figure 4. Spatial organization of a dense dune field with $\xi=2.9$ and $L=32$. (a) Snapshot of a computed desert. Colors represent the dune size. (b) Close-up of a cluster of Figure 4a. (c) Transverse profiles of local mean width $\langle w\rangle_{l}$ and local number $N_{l}$ of dunes of the surrounded region of the field (Figure $4 \mathrm{a}$ ). $\langle w\rangle_{l}$ and $N_{l}$ show anticorrelation, see dashed and dash-dotted lines, for example. (d) Local mean width $\langle w\rangle_{l}$ as a function of the local dune density $\rho_{l} .\langle w\rangle_{l}$ and $\rho_{l}$ are computed for boxes of size $w_{0} \times w_{0}$. Values are averaged over the whole field and over a long period of time. Error bars stand for standard deviation. The size of dunes is smaller and better selected as the density increases. Movies showing the dynamics of barchan fields are available as supporting information. 

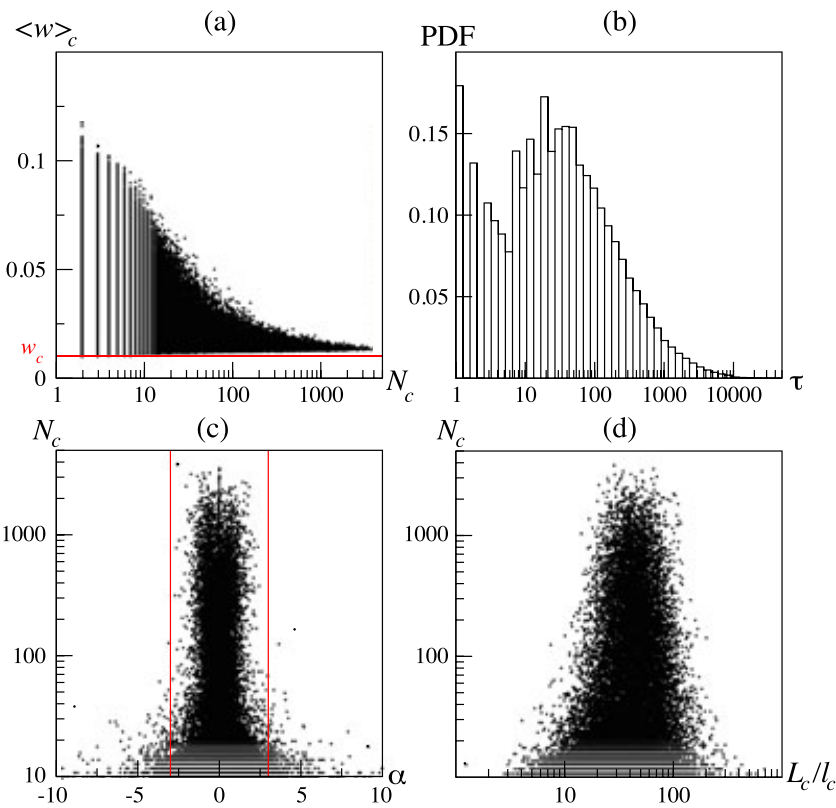

Figure 5. Some properties of clusters in a dense regime, $\xi=2.9$ and $L=32$. (a) Mean size of dunes $\langle w\rangle_{c}$ in a cluster versus the number $N_{c}$ of dunes of the same cluster. The red line marks the size of vanishing dunes $w_{c}$. (b) Distribution of the cluster lifetime. (c) Number $N_{c}$ of dunes in a cluster versus the angle between the main axis of the same cluster and the wind direction. The two red lines show an amplitude of $6^{\circ}$, which corresponds to five standard deviations. (d) Number $N_{c}$ of dunes in a cluster versus the aspect ratio of the same cluster.

collisions. On the contrary, dense spatial clusters of small interacting dunes (i.e., with interdunes distance smaller than $d_{0}$ ) develop and gather most of the dunes when $\xi$ increases (Figure 3d). As shown in Figures 4 and 5, these clusters have sharp boundaries and are elongated along the wind direction. Thus, the field is self-structured in a corridor-like pattern where the local density is a highly fluctuating quantity. If we restrict our measurements to local low density, the dune size distribution shows a maximum at $w_{0}$ as in a dilute desert. The local dune size is directly correlated to the local density of dunes and decreases when this latter increases (Figure 4). A dense field looks like a dilute field of big dunes with dense corridors of small dunes. These spatial structures and relation between dune size and dilution are similar to what is observed in the long barchan field that extends in the Atlantic Sahara (Morocco), see Elbelrhiti et al. [2008] and Figure 1.

[16] The formation of corridors in dense dune fields is robust and to that respect, observed for a range of the three characteristic times $t_{\mathrm{eol}}, t_{\mathrm{nuc}}$, and $t_{\mathrm{col}}$ when fragmenting collisions are allowed, i.e., when $\epsilon_{s}>0$. These results demonstrate that a dune fragmentation mechanism, which seems to lack in previous studies, is a key process in setting the dune size but also in the emergence of dense barchan corridors within a dune field. Here the fragmentation mechanism is provided by collisions but one can think of other barchan destabilization mechanism such as calving of small dunes from the arms of big barchans [Elbelrhiti et al., 2005; Zhang et al., 2009]. However, the selection of size and the blowup of the number of dunes in corridors result from a dynamical effect. Collisions produce small dunes that go faster and collide downwind dunes in an avalanche-like process (Figure 5a). Note that these small dunes would have a very limited lifetime if isolated but that they do not lose sand in a corridor where all dunes exchange sand and sustain each other. A corridor loses sand only at its downwind boundary.

[17] Transitory times are also very different between dilute and dense deserts. Whereas the number of dunes relaxes normally in dilute deserts, dense deserts exhibit nearly periodic blowup of their population (not shown here). Therefore, cluster properties are not Gaussian statistical fluctuations but are reinforced by dune-to-dune interaction. This behavior is consistent with a process of phase transition. However, the finite lifetime of clusters (Figure 5b) finally inhibits any long range order that could appear at a phase transition.

[18] Increasing $\xi$ further, both the number of clusters and cluster size increase (Figure 3 ). The dunes are smaller, corridors are more aligned in the wind direction, and their aspect tends to a line (about 40 times longer than wide). However, note that our model may not be relevant for very high sand density for which barchans may laterally link into barchanoid ridges and eventually form transverse dunes [Wasson and Hyde, 1983; Kocurek et al., 2010]. Such a mechanism is not included in our model. Indeed, although transverse dunes are known to be unstable on a nonerodible floor when there is no sand supply [Reffet et al., 2010; Parteli et al., 2011; Guignier et al., 2013], they might be stabilized by a sand input. The dune field might also not reach stationary state then.

\section{Conclusion}

[19] We introduced a minimal agent-based model of barchans in interactions, in which kinematics and interactions are set in considering experimental evidence of dune collisions. Its domain of validity cannot extend outside the framework of statistical physics: infinite size and infinite time of observations. However, varying the lifetime of barchans by tuning the sand loss, we showed a smooth crossover from dilute deserts to dense ones where dunes aggregate in elongated clusters. The computed deserts selforganize in corridor-like patterns where dense regions are composed by small dunes, and dilute spaces show larger dunes. This is observed in dense barchan fields on Earth. Our model, although minimal, was able to capture the emergence of such heterogeneous patterning. We demonstrated in particular that the fragmenting collision between barchans, which is a dynamical effect that produces dunes in cascade, is a mechanism that can explain the formation of dense corridors of barchan with a small, well-selected size. One can now think to add complexity to even better represent realworld scenarios. In particular, it would be interesting to add an ambient influx, to limit the barchan sand loss to the arms only, and to consider the long range remote sand exchange. Experiments and numerical studies would also be fruitful to better constrain the phase diagram of collisions, the linking of barchans into transverse dunes, and the respective breaking, inverse transition.

[20] Acknowledgments. Reviews by D. Jerolmack and an anonymous reviewer were valuable. S. Courrech du Pont is supported by Agence Nationale de la Recherche grant ANR 12 BS05 001 02/EXO-DUNES.

[21] The Editor thanks two anonymous reviewers for assistance evaluating this manuscript. 


\section{GÉNOIS ET AL.: WHEN DUNES MOVE TOGETHER}

\section{References}

Bagnold, R. A. (1941), The Physics of Blown Sand and Desert Dunes, Chapman and Hall, London.

Bourke, M., and A. Goudie (2009), Varieties of barchan form in the Namib Desert and on Mars, Aeolian Res., 1(1-2), 45-54, doi:10.1016/ j.aeolia.2009.05.002.

Breed, C. S., M. J. Grolier, and J. F. McCauley (1979), Morphology and distribution of common "sand" dunes on Mars: Comparison with the Earth, J. Geophys. Res., 84 (B14), 8183-8204, doi:10.1029/ JB084iB14p08183.

Chaté, H., F. Ginelli, G. Grégoire, and F. Raynaud (2008), Modeling collective motion: Variations on the Vicsek model, Eur. Phys. J. B, 64, 451, doi:10.1140/epjb/e2008-00275-9.

Cooke, R. U., A. Warren, and A. S. Goudie (1993), Desert Geomorphology, UCL Press Limited, University College London, London, U. K.

Devauchelle, O., A. P. Petroff, H. F. Seybold, and D. H. Rothman (2012), Ramification of stream networks, PNAS, 109 (51), 20,832-20,836, doi:10.1073/pnas. 1215218109 .

Diniega, S., K. Glasner, and S. Byrne (2010), Long-time evolution of models of aeolian sand dune fields: Influence of dune formation and collision, Geomorphology, 121, 55-68, doi:10.1016/ j.geomorph.2009.02.010.

Dodds, P., and D. Rothman (2000), Scaling, universality, and geomorphology, Annu. Rev. Earth Planet. Sci., 28(1), 571-610, doi:10.1146/annurev. earth.28.1.571.

Durán, O., V. Schwammle, and P. G. Lind (2009), The dune size distribution and scaling relations of barchan dune fields, Granular Matter, 11(1), 7-11, doi:10.1007/s10035-008-0120-4.

Durán, O., V. Schwammle, P. G. Lind, and H. J. Herrman (2011), Size distribution and structure of barchan dune fields, Nonlinear Processes Geophys., 18(4), 455-467, doi:10.5194/npg-18-455-2011.

Elbelrhiti, H., P. Claudin, and B. Andreotti (2005), Field evidence for surface-wave-induced instability of sand dunes, Nature, 437, 720-723, doi:10.1038/nature04058.

Elbelrhiti, H., B. Andreotti, and P. Claudin (2008), Barchan dune corridors: Field characterization and investigation of control parameter, J. Geophys. Res., 113, F02S15, doi:10.1029/2007JF000767.

Endo, N., Taniguchi K., and Katsuki A. (2004), Observation of the whole process of interaction between barchans by flume experiments, Geophys. Res. Lett, 31, L12503, doi:10.1029/ 2004GL020168.

Finkel, H. (1959), The barchans of southern Peru, J. Geol., 67, 614-647.

Guignier, L., H. Niiya, H. Nishimori, D. Lague, and A. Valance (2013), Sand dunes as migrating strings, Phys. Rev. E, 87, 052206, doi:10.1103/PhysRevE.87.052206.

Hersen, P. (2004), On the crescentic shape of barchan dune, Eur. Phys. J. B, 37, 507-514, doi:10.1140/epjb/e2004-00087-y.
Hersen, P., and S. Douady (2005), Collision of barchan dunes as a mechanism of size regulation, Geophys. Res. Lett., 32, L21403, doi:10.1029/2005GL024179.

Hersen, P., K. H. Andersen, H. Elbelrhiti, B. Andreotti, P. Claudin, and S. Douady (2004), Corridors of barchan dunes: Stability and size selection, Phys. Rev. E, 69(1), 011-304, doi:10.1103/PhysRevE. 69.011304.

Hugenbholtz, C., and T. Barchyn (2012), Real barchan dune collisions and ejections, Geophys. Res. Lett., 39, L02306, doi:10.1029/ 2011 GL050299.

Katsuki, A., N. Endo, and K. Taniguchi (2005), Collision dynamics of two barchan dunes simulated by a simple model, J. Phys. Soc. Japan, 37 , 507-514, doi:10.1143/JPSJ.74.538.

Kocurek, G., R. Ewing, and D. Mohrig (2010), How do bed form patterns arise? New views on the role of bed form interactions within a set of boundary conditions, ESPL, 35(1), 51-63, doi:10.1002/esp.1913.

Kroy, K., G. Sauermann, and H. J. Herrmann (2002), Minimal model for aeolian sand dunes, Phys. Rev. E, 66, 031-302, doi:10.1103/ PhysRevE.66.031302.

Lima, A., H. Sauermann, H. Herrmann, and K. Kroy (2002), Modelling a dune field, Physica A, 310, 487-500, doi:10.1016/S03784371(02)00546-0.

Parteli, E., J. Andrade, and H. Herrmann (2011), Transverse instability of dunes, Phys. Rev. Lett, 107(188), 001, doi:10.1103/PhysRevLett. 107.188001.

Pye, K., and H. Tsoar (1990), Aeolian Sand and Sand Dunes, Unwin Hyman, London.

Radebaugh, J., R. Lorenz, T. Farr, P. Paillou, C. Savage, and C. Spencer (2010), Linear dunes on Titan and Earth: Initial remote sensing comparisons, Geomorphology, 121(1-2), 122-132, doi:10.1016/ j.geomorph.2009.02.022.

Reffet, E., S. Courrech du Pont, P. Hersen, and S. Douady (2010), Formation and stability of transverse and longitudinal sand dunes, Geology, 39(6), 491-494, doi:10.1130/G30894.1.

Rodriguez-Iturbe, I., and A. Rinaldo (1997), Fractal River Basins: Chance and Self-Organization, Cambridge Univ. Press, Cambridgre, U.K.

Schwämmle, V., and H. J. Herrmann (2003), Solitary wave behaviour of sand dunes, Nature, 426, 619-620, doi:10.1038/426619a.

Vermeesch, P. (2011), Solitary wave behavior in sand dunes observed from space, Geophys. Res. Lett., 38, L22, doi:10.1029/2011GL049610.

Vicsek, T., A. Czirok, E. Benjacob, I. Cohen, and O. Shochet (1995), Novel type of phase-transition in a system of self-driven particles, Phys. Rev. Lett, 75(6), 1226-1229, doi:10.1103/PhysRevLett.75.1226.

Wasson, R., and R. Hyde (1983), Factors determining desert dune type, Nature, 304, 337-339, doi:10.1038/304337a0.

Zhang, D., C. Narteau, and O. Rozier (2009), Morphodynamics of barchan and transverse dunes using a cellular automaton model, J. Geophys. Res., 115, F03041, doi:10.1029/2009JF001620. 